\title{
Reviewing HIV-1 Gag Mutations in Protease Inhibitors Resistance: Insights for Possible Novel Gag Inhibitor Designs
}

Chinh Tran-To Su${ }^{1}$, Darius Wen-Shuo Koh ${ }^{1}$, Samuel Ken-En Gan ${ }^{1,2 *}$

${ }^{1}$ Antibody \& Product Development Lab, Bioinformatics Institute, A*STAR, Singapore, 138671 ${ }^{2}$ p53 Laboratory, A*STAR, Singapore 138648

${ }^{*}$ Corresponding author:

Samuel Ken-En Gan

samuelg@bii.a-star.edu.sg

Tel: +6564788417

Fax: +6564789047

Running title: Prospects of Gag inhibitor design and use 


\section{ABSTRACT}

HIV protease inhibitors against the viral protease are often hampered by drug resistant mutations that are found in protease and in the viral substrate Gag. To overcome this drug resistance and inhibit viral maturation, it may be more efficient to target Gag alongside protease than targeting protease alone. In order to successfully inhibit Gag, understanding of its drug resistance mutations and the elicited structural changes on protease binding needs to be investigated. While mutations on Gag have already been mapped to resistance to protease inhibitors, there remain many mutations, particularly the non-cleavage mutations, that are not characterized. Through structural studies to unravel how Gag mutations contributes to protease drug resistance synergistically, it is thus possible to glean insights to design novel Gag inhibitors. In this review, we discuss the structural role of both novel and previously reported Gag mutations in PI resistance, and how new Gag inhibitors can be designed.

Keywords: HIV-1 Gag; Gag inhibitors; Protease; Protease inhibitors; drug resistance mutations; drug design 


\section{Introduction}

Many anti-HIV drugs interfere directly with the viral life cycle by targeting key viral enzymes [1], e.g. reverse transcriptase inhibitors [2,3], integrase inhibitors [4,5], and protease inhibitors $[6,7]$. While such efforts are already hampered by the emergence of drug resistance mutations in the enzymes (e.g. reported in [8]), the scenario further worsens when viral enzyme substrates, such as Gag (HIV protease substrate), are found to contribute to drug resistance. Gag and protease play key roles in the viral maturation process [9] where the immature HIV virion matures into the infectious virion after budding from the infected cell for the next replication cycle. Proteolysis of Gag by protease occurs during the early stage of this maturation (Figure 1A), in which the intact full length Gag precursor polyprotein is cleaved by the viral protease into functional subunits [9]. To inhibit this proteolysis, Protease Inhibitors (PIs) block protease activity in a competitive manner with Gag for protease binding [10].

PI-resistant mutations have been reported on Protease [11-13] and Gag [14-18] alone, or concurrently on both Protease and Gag [17,19-22], revealing an enzyme-substrate synergy to overcome PIs [23] (Figure 1B). Expectedly, Gag cleavage site mutations contribute directly to PI resistance [24], while non-cleavage site mutations contribute to drug resistance by compensating for the loss of viral fitness $[22,25,26]$ resulted when protease accumulates drug resistant mutations that reduced its proteolytic functions.

As Gag is a larger protein, and mutations (both cleavage and non-cleavage) can contribute to $\mathrm{PI}$ resistance, there is thus a need to study the mechanisms to how these mutations work in synergy with protease. Such studies will unravel potential weak points to which Gag can be targeted against, opening more opportunities in drug design approach. 


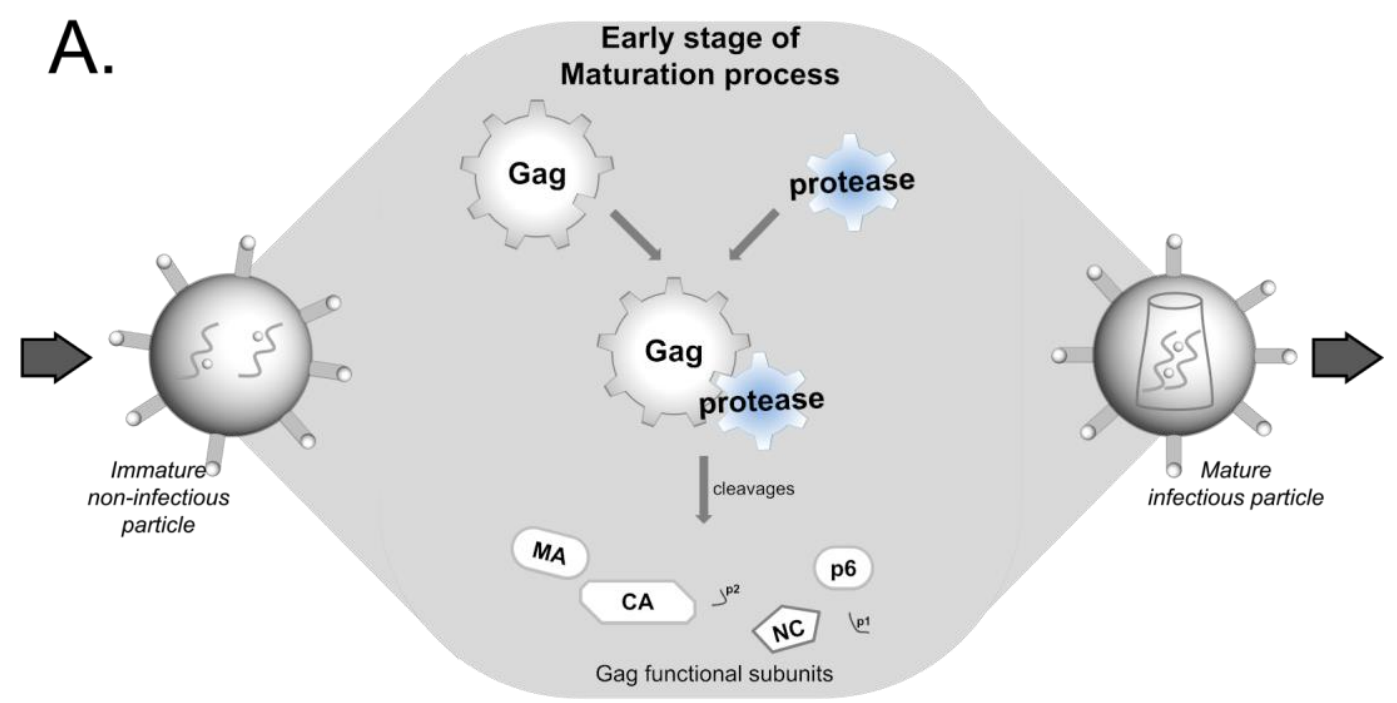

B.
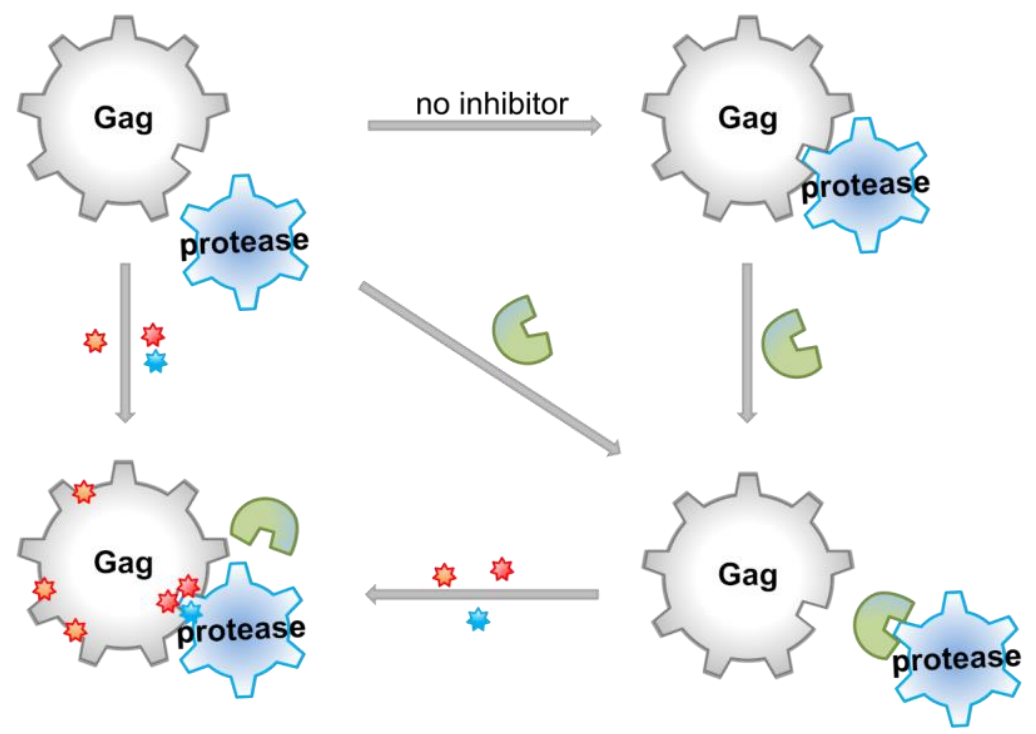

Figure 1: Overview of Gag and Protease relationship. (A) Schematic of the early stage of viral maturation where HIV-1 Protease cleaves Gag into the functional subunits: matrix (MA), capsid (CA), nucleocapsid (NC), p6 and two spacer peptides p1 and p2. (B) To inhibit viral maturation, Protease Inhibitors (Pls in green) is used to competitively inhibit protease binding of Gag. PI resistant mutations are denoted by colored stars, where those in the protease catalytic site are in blue, while those in the Gag, are red for cleavage sites, and orange for non-cleavage sites.

\section{Possible Targets in Gag}

The Gag polyprotein consists of components matrix (MA), capsid (CA), nucleocapsid (NC), p6, and two spacer peptides $\mathrm{p} 1$ and $\mathrm{p} 2$. The MA subunit, located at the $\mathrm{N}$-terminus, is essential for targeting Gag to the cell membrane, while the CA forms a shell to protect the viral RNA 
genome and other core proteins during maturation. The NC is responsible for RNA packing and encapsidation [27] while the two spacer peptides $\mathrm{p} 1$ and $\mathrm{p} 2$ regulate the rate and the sequential cleavage process of Gag by protease [28]. This process of viral assembly is complemented by viral budding moderated by the small Proline-rich p6. Mutations at either the $\mathrm{N}$-terminal or $\mathrm{C}$-terminal of these core proteins were reported to block viral assembly and impair Gag binding to plasma membrane, thereby inhibiting viral budding [27].

Since the Gag cleavage sites did not share a consensus sequence (Figure 2), the recognition of the cleavage sites by protease is likely to be based on their asymmetric 3-dimensional structures [29] that would fit into the substrate-binding pocket of protease [30]. The cleavage of these scissile bonds (7-residue peptide sequences unique for each cleavage site) are highly regulated and occur at differing rates $[24,28,31]$. The first cleavage occurs at the site between the p2 peptide and NC domain (Figure 2), followed by the MA from CA-p2 at a rate that is 14fold slower than that of the first cleavage, before proceeding to release $p 6$ from the NC-p1 domain (at a rate $\sim 9$-fold slower than the first cleavage). Finally the two spacer peptides $\mathrm{p} 1$ and p2 are cleaved from NC-p1 and CA-p2 at rates $\sim 350$-fold and $\sim 400$-fold, respectively, slower than the initial cleavage $[24,28,30,31]$.

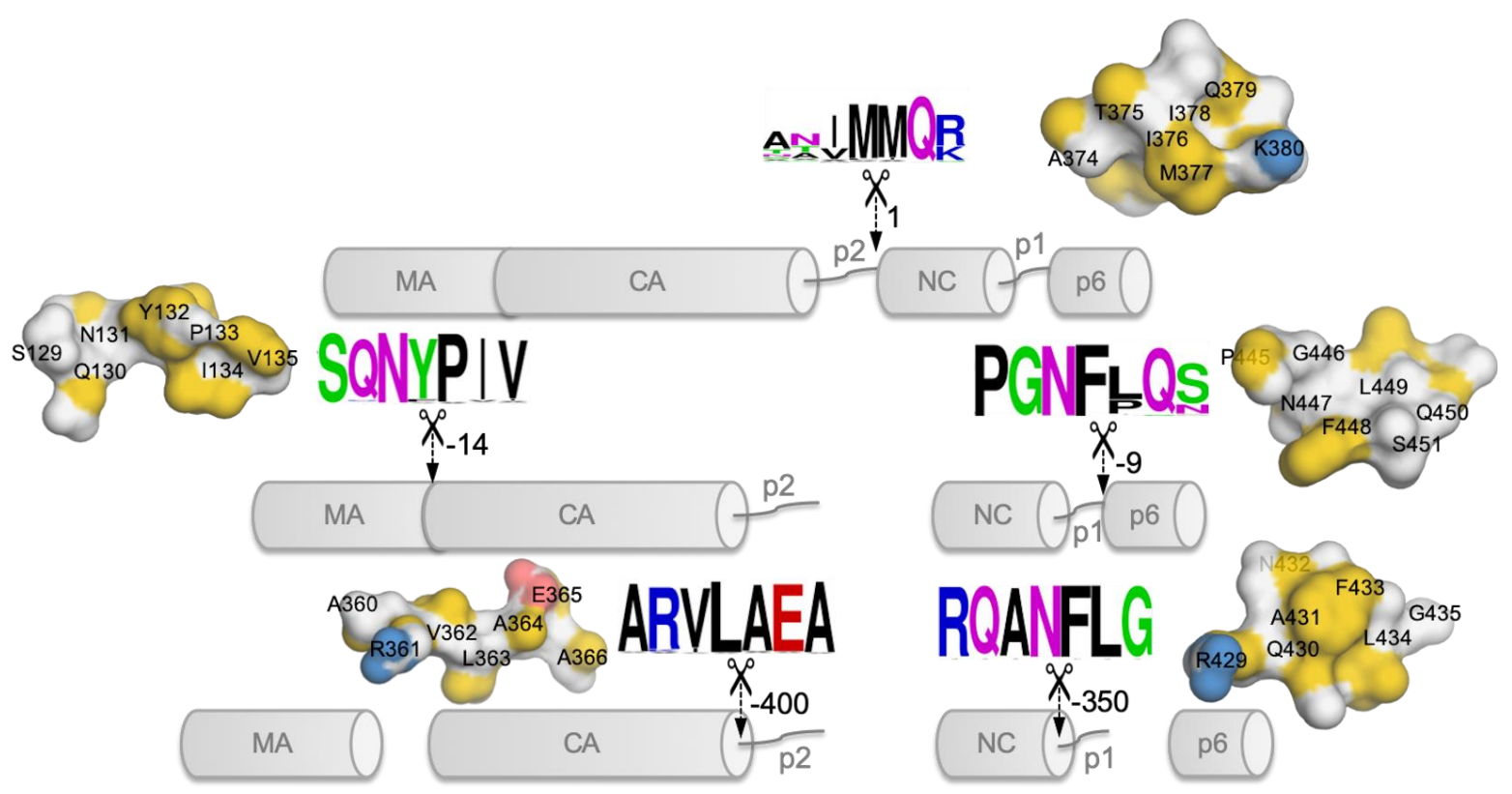

Figure 2: The sequential Gag proteolysis by Protease. The cleavage sites are marked by the 7-residues, along with the estimated cleavage rates [28] marked by arrows. For easy comparison, the initial cleavage site rate is set to the value of 1 , while the other cleavage site 
values depict the reduced normalized rate. The cleavage site sequences are colored based on their physicochemical properties, e.g. hydrophobic (black), charged (positive: blue, negative: red), polar (other colors) and varied in text sizes based on positional conservation, using WebLogo [32,33]. Structural surface presentations of the cleavage sites are also attached for visualization.

To date, there are nine Pls, i.e. Saquinavir (SQV), Ritonavir (RTV), Indinavir (IDV), Nelfinavir (NFV), Fos/Amprenavir (FPV/APV), Lopinavir (LPV), Atazanavir (ATV), Tipranavir (TPV), and Darunavir (DRV) in clinical treatment [30]. With increasing PI resistance [34-37] and crossresistance $[21,24,35,38]$ conferred by protease mutations that compromise viral fitness, there is a compromise between enzymatic activity and drug inhibition by protease within its 99residue homodimer subunits. Mapped to resistance to several current Pls [39-42], many mutations were found to spontaneously arise as part of the natural variance [43] that were selected for during the treatment regimes. These mutations directly intervene with PI binding via steric perturbation at the active site, and those distant from the active site, allosterically modulated protease activity $[12,13,44-52]$. However, such mutations often reduce viral fitness, resulting in future repertoires of compromised fitness viruses [53]. Particularly, this fitness trade-off is then compensated by additional mutations that restore enzymatic activity to an extent $[44,48,49,54]$.

Reports of Gag PI-resistant mutations [17,19-22,24], whether independent or linked to protease mutations, include those that restore the reduced binding affinity to mutated proteases $[17,19$ $24,55,56]$. Such mutations were reported to be found throughout the whole Gag structure with the majority on MA and p6 domains, playing a major role towards therapy failure $[15,23]$. In fact, multiple Gag inhibitors were rendered ineffective due to natural Gag polymorphisms [57]. New clinical protease resistant mutations are decreasingly reported, hinting a limit of the mutations tolerable within protease. On the other hand, with $\sim 500$ residues, Gag has more leeway for compensations in the Gag-protease synergy towards drug resistance. However, when compared to protease, Gag is comparatively neglected, lacking a dedicated curated database (e.g. protease in the Los Alamos and Stanford HIV databases). 
To fully study the Gag-protease synergy, there is a need to study the limitations and mechanisms by which Gag mutations arise. Although the sequencing clinical samples is the predominant source of HIV sequences, there have been attempts to study and generate novel mutations (see preprint [58] and [59,60]) for various HIV proteins. One example of such an effort [58] involved subjecting the Gag mRNA transcript to HIV reverse transcriptase (RT) to explore the repertoire of possible Gag mutations in the absence of drug or immune selection pressures. It was shown that clinically reported mutations could be generated and that the location and type of mutations generally avoided crucial locations and drastic changes. While such selection-free platforms can reveal the possible repertoires of Gag mutations for inhibitor design against emerging resistance, the large permutations require focusing through structural analysis for comparison to known clinical mutations.

Characterized clinical Gag mutations [17,19-22,24] are sparse, with many reported to restore reduced binding to mutated proteases $[17,19-24,55,56]$. The lack of a high-resolution structure of full-length Gag for study of these mutations makes it difficult to analyze structurally the effects of these mutations on the whole Gag during its binding to protease. The full length model of Gag [61] recently allowed the investigation of non-cleavage site mutations on the first cleavage site. Coupling generated mutations for study in the full Gag model, it is possible to investigate the effects of the mutations before they are clinically observed. Yet, for the design of inhibitors to the remaining Gag cleavage sites or study the effects of all the non-cleavage site mutations, structures of all the Gag subunits at every cleavage step are necessary.

\section{The role of Gag mutations in restoring Gag-Protease synergy in PI resistance}

The mapping of Gag mutations associated with protease drug resistant mutations are summarized in Table 1. Gag cleavage site mutations at the p1/p6 (L449F) and NC/p1 (L449FQ430R-A431V) sections were found to be associated to protease mutation I84V [24,62]. Similarly, Gag mutations A431V and I437V were mapped to protease mutation V82A $[24,63]$. 
Apart from compensating the loss of viral fitness, mutations P453L (Gag) and I50V (Protease) synergistically mitigated Amprenavir effectiveness (e.g. increasing $I_{50}$ value of Amprenavir), and Gag mutations A431V-I437V together with protease V82A were found to lead to Indinavir resistance [24].

Non-cleavage site mutations associated with PI resistance [18,22], included H219Q and R409K for Amprenavir, JE-2147, KNI-272, and UIC-94003 resistance. Gag L75R and H219Q together with Protease mutation I84V, led to Amprenavir and JE-2147 resistance. Together, these non-cleavage site mutations (synergistically with E12K, V390D, and R409K) delayed resistance to other Pls e.g. Ritonavir and Nelfinavir [18]. Interestingly, most of these Gag noncleavage site mutations are located on the MA-CA or p1-p6 domains. Gag MA domain mutations (e.g. R76K, Y79F, and T81A) were suggested to enhance Protease accessibility to Gag cleavage sites [15,64]. Nonetheless, the exact mechanism of such non-cleavage mutations remains elusive due to the lack of full-length Gag structure and its sequentially cleaved subunits.

Structural research $[45,47,61,65]$ have revealed an underlying allosteric mechanism in resistance development by Gag non-cleavage mutations that allosterically rendered the first cleavage site to be more flexible [61]. When coupled with protease mutations, several Gag compensatory mutations recovered protease binding affinities. Thus, the Gag and protease mutations synergistically formed a resistance network against multiple Pls $[39,65,66]$. By mapping these Gag-protease resistance relationship (Figure 3) onto our previously constructed PI cross-resistance network [65], similar combinations of Gag mutations were found to resist varied PIs, independent on their diverse chemical scaffolds [67]. 


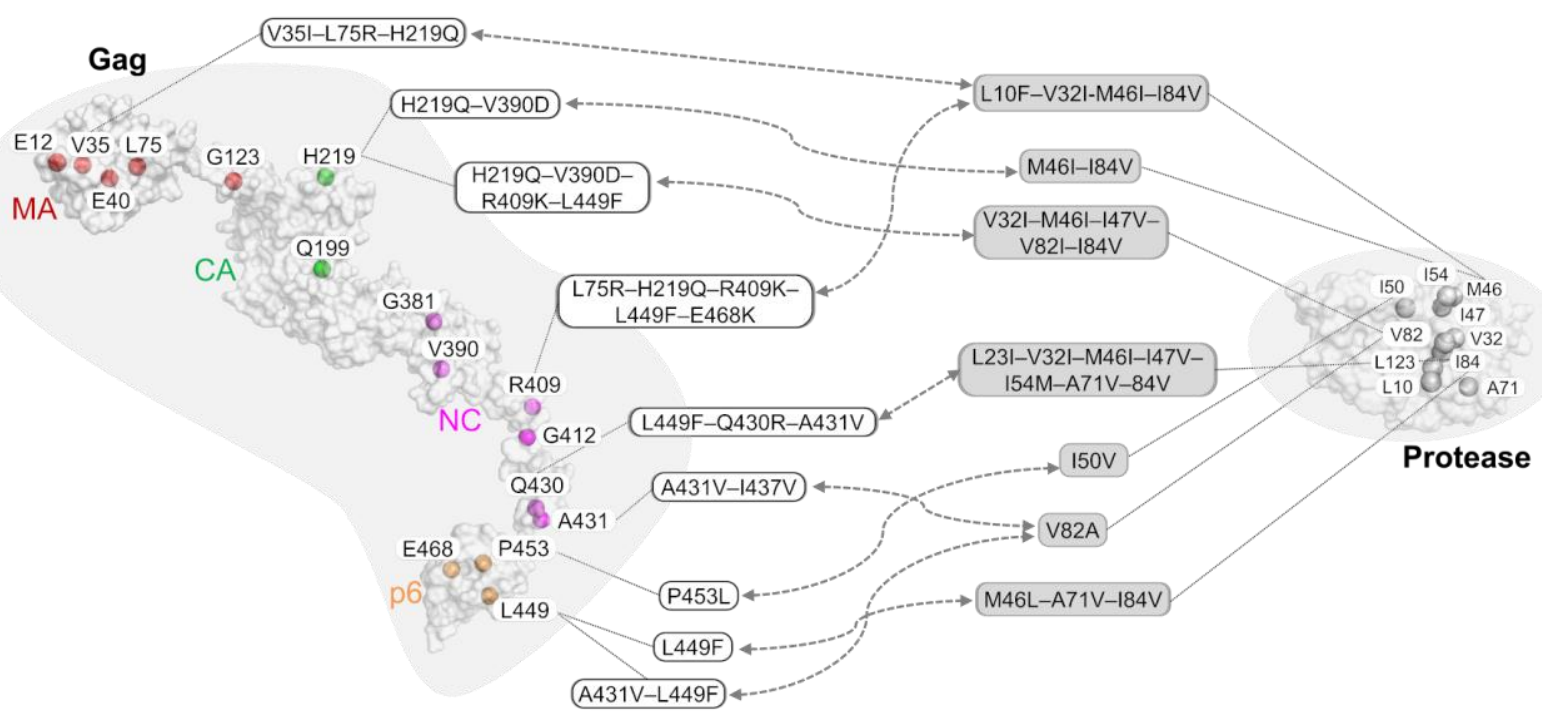

Figure 3: A schematic map of associated Gag-Protease drug resistant mutations. Mutation hotspots are shown on both the Gag and Protease, and representatives of paired combinations of $\mathrm{Gag}$ and Protease mutations are shown in boxes. More details can be found in Table 1. Gag mutations are colored according to domains: MA (red), CA (green), NC (magenta), and p6 (orange).

\section{Conceptual novel designs of Gag Inhibitors}

Current drugs used in the ART treatment are cocktails of PIs, NRTIs, and NNRTIs [42,68-70].

Others treatment regimens can involve therapeutic antibodies that target viral proteins [71-73] or Gag epitopes [74]. Among the maturation inhibitors, Gag inhibitors have faced significant challenges. Berivimat (Panacos PA-457, Myriad MPC-4326) is the first Gag inhibitor targeting the CA-p2 cleavage site for Gag assembly [75-79] to undergo clinical trials (refer to supplementary material of [57]). Yet, Gag polymorphisms $[80,81]$ and accumulated resistant mutations during PI exposure $[82,83]$ rendered the inhibitor candidate ineffective. Nevertheless, second-generation maturation inhibitors based on Berivimat continue to be developed and explored $[84,85]$.

Given that the maturation process takes place within the closed environments inside immature virions or intracellularly, large biologics are less likely than small molecule inhibitors to be successful. Since Gag non-cleavage resistance mutations are found across the Gag structure (Figure 3), novel Gag inhibitors can inhibit Gag allosterically via its domain cross-talks $[61,86]$. 
With sequentially cleaved Gag structures, we propose four approaches to design Gag inhibitors (Figure 4) while keeping a holistic view of the protein as previously discussed [86]. The first approach, stems from a holistic view to computationally screen the whole Gag structure for novel allosteric druggable pockets [86] to (i) inhibit compensation effects of noncleavage mutations or (ii) directly influence cleavage sites to block protease. While the Gag cleavage sites vary in sequences, their structural similarities may allow for druggable shared allosteric sites (see example of druggable allosteric pocket in HIV-1 Reverse Transcriptase [87]). Alternatively, a "multi-cleavage site" inhibitor would target the multiple cleavage sites directly (e.g. by targeting the common hydrophobic residue at the $5^{\text {th }}$ position of the 7sequence peptide, Figure 2) or indirectly via targeting multiple druggable allosteric pockets given the structural recognition. An example of such a "broad-spectrum" structural allosteric inhibitor that could inhibit HIV-1 and the Moloney murine leukemia virus (MMLV) reverse transcriptase (unpublished, see preprint [88]) was found by structural similarity of the binding site. In fact, aiming multiple targets would also delay resistance since the possibility of all the target sites gaining drug resistance simultaneously is lower.

The second is a preemptive approach. By generating Gag mutations to investigate possible emerging Gag mutants (see example where Gag gene was reverse transcribed by HIV RT to generate novel mutations in unpublished preprint [58]), novel mutations can be identified and modelled. Peptidomimetics in pre-emptive inhibitors of emerging resistant variants can bottleneck Gag towards eradication. Such inhibitors can include the allosteric or multiple site inhibitors, and given the wide range of possibilities, it would certainly require in-depth structural analysis to limit the permutations and combinations of mutations.

The third approach is to chemically join different inhibitors of (i) Gag cleavage inhibitors; (ii) Gag allosteric inhibitors (either to cleavage sites or to inhibit compensatory effects); and (iii) existing PIs. Such compounds can function as dual/triple inhibitors (Figure 4) that are localized to the area of Gag-protease activity when already bound to a target. If coupled to PIs, such dual/triple inhibitors mimic antibodies and can result in cytosolic aggregation of Gag/protease complexes for degradation or host cell death. The use of synergistic drugs that target multiple 
sites have been shown promising in cancer treatment, e.g. using combined biologics Trastuzumab and Pertuzumab antibodies (both of which target different epitopes on the same cancer marker Her2) with marked clinical improvements [89,90].

The fourth and the last approach is to disrupt Gag conformational transition during viral maturation, i.e. "throwing a wrench into a running gear" (Figure 4). Since p6 could perturb MACA or NC motions when Gag was compact and modulate structural stability of these Gag conformations [61], the "gear" (Gag changing conformation) could be jammed, thus destabilizing Gag assembly. Such a "wrench" could bind at least two conserved sites in Gag to constrain Gag conformation. Potential binding sites can be the conserved regions of NC and $\mathrm{p} 6$ to rigidify $\mathrm{p} 6$ and perturb the allosteric signaling to the MA-CA region [61] to also interfere with required Gag oligomerization [91-93]. By preventing conformational change from the compact to the extended structure, the exposure of subsequent cleavage sites could be reduced, reducing the viral fitness by slowing down the viral maturation process. 
I. Screening \& targeting allosteric sites

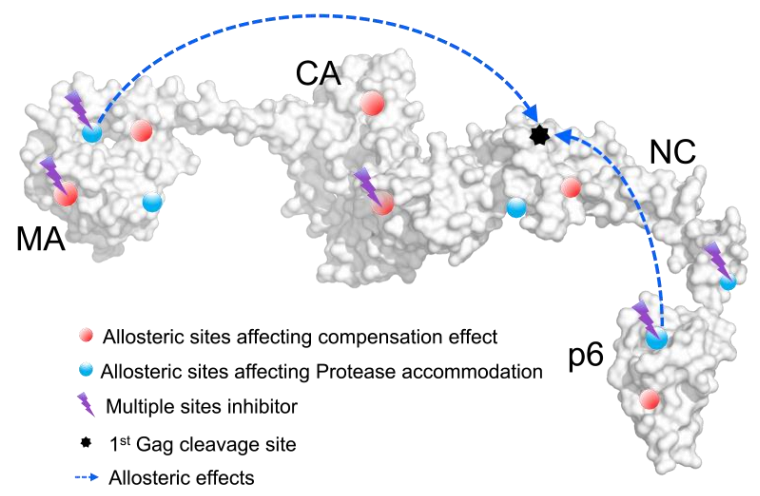

III. Dual inhibitor

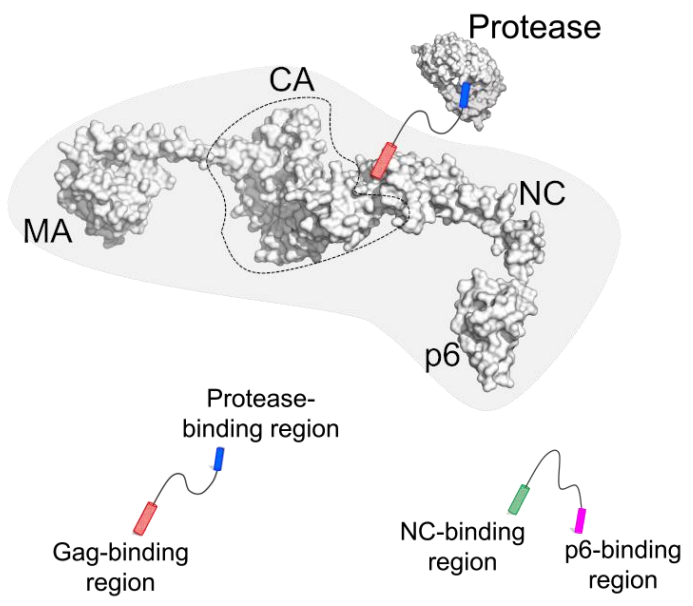

II. An preemptive approach

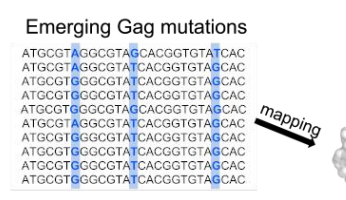

* Substitution mutation

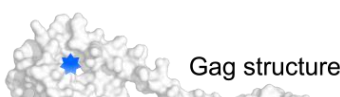

Gag structure

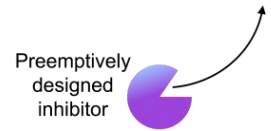

IV. Gag conformational constrainer

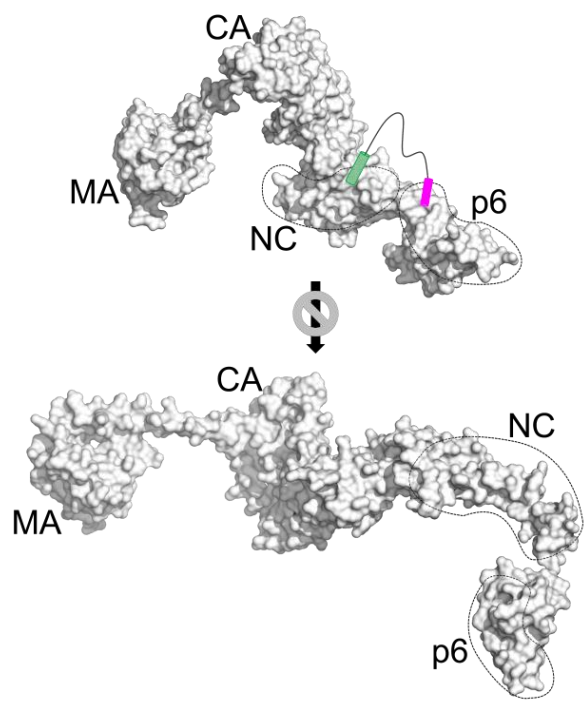

Figure 4: The four proposed conceptual designs of Gag-inhibitors.

\section{CONCLUSION AND FUTURE PERSPECTIVE}

The increasing prominence of Gag mutations in PI resistance calls for new strategies in inhibiting HIV maturation. While there are reports of Gag resistant mutations and a full-length Gag model, much remains to be studied. There is a need to characterize Gag mutations in a curated annotated database for documenting their effects. At the same time, structures/models of Gag subunits of the various sequential cleavages allow the study of documented or potential emerging Gag mutations.

Better structural and generation of emerging mutations in Gag would give rise to new types of HIV-1 drug candidates in the clinic for the next decades. These new candidates are likely to 
complement the current ART to form a tighter bottleneck to reduce viral load. Although it is still too early to speculate whether the Gag inhibitor candidates are likely to be small molecules or peptide inhibitors, it is reasonable to assume that they would be designed based on the structural information of the Gag cleavage sites and would primarily act to prevent protease binding or disrupt Gag assembly. Equipped with the understanding of how these non-cleavage mutations in Gag compensate viral fitness, there would be more alternative mechanisms to target for Gag inhibitors.

\section{Acknowledgement}

The work is supported by core fund of the Antibody and Product Development (APD) Lab, Bioinformatics Institute, $A^{\star}$ STAR.

\section{Author Contributions}

All authors conceived ideas and wrote the manuscript. All authors read and approve it.

\section{Conflicts of interest}

Authors declare no conflict of interest. 


\section{REFERENCES}

1. Bhatti, A.B.; Usman, M.; Kandi, V. Current scenario of HIV/AIDS, treatment options, and major challenges with compliance to antiretroviral therapy. Cureus 2016, 8, e515.

2. Rai, M.; Pannek, S.; Fichtenbaum, C. Emerging reverse transcriptase inhibitors for HIV-1 infrection. Expert Opin. Emerg. Drugs 2018, 23, 149-157.

3. Orkin, C.; Llibre, J.; Gallien, S.; Antinori, A.; Behrens, G.; Carr, A. Nucleoside reverse transcriptase inhibitor-reducing strategies in HIV treatment: assessing the evidence. HIV Medicine 2017, 19, 18-32, doi:10.1111/hiv.12534.

4. Debyser, Z.; Vansant, G.; Bruggemans, A.; Janssens, J.; Christ, F. Insight in HIV Integration Site Selection Provides a Block-and-Lock Strategy for a Functional Cure of HIV Infection. Viruses 2019, 11, 12, doi:10.3390/v11010012.

5. Charpentier, C.; Descamps, D. Resistance to HIV Integrase Inhibitors: About R263K and E157Q mutations. Viruses 2018, 10, 41, doi:10.3390/v10010041.

6. Favarato, G.; Townsend, C.L.; Bailey, H.; Peters, H.; Tookey, P.A.; Taylor, G.P.; Thorne, C. Protease inhibitors and preterm delivery: another piece in the puzzle. AIDS 2018, 32, 243-252, doi:10.1097/QAD.0000000000001694.

7. Lv, Z.; Chu, Y.; Wang, Y. HIV Protease inhibitors: a review of molecular selectivity and toxicity. HIV AIDS (AuckI) 2015, 7, 95-104, doi:10.2147/HIV.S79956.

8. $\quad$ Castain, L.; Perrier, M.; Charpentier, C.; Palich, R.; Desire, N.; Wirden, M.; Descamps, D.; Sayon, S.; Landman, R.; Valantin, M.-A., et al. New machanism of resistance in virological failure to protease inhibitors: selection of non-described protease, Gag and Gp41 mutations. Journal of Antimicrobial Chemotherapy 2019, 74, 2019-2023, doi:10.1093/jac/dkz151.

9. Freed, E.O. HIV-1 assembly, release and maturation. Nature Reviews 2015, 13, 484496, doi:10.1038/nrmicro3490.

10. Ghosh, A.K.; Osswald, H.L.; Prato, G. Recent progress in the development of HIV-1 Protease Inhibitors for the treatment of HIV/AIDS. Journal of Medicinal Chemistry 2016, 59, 5172-5208, doi:10.1021/acs.jmedchem.5b01697.

11. Wensing, A.M.; Calvez, V.; Günthard, H.F.; Johnson, V.A.; Paredes, R.; Pillay, D.; Shafer, R.W.; Richman, D.D. 2017 Update of the Drug Resistance Mutations in HIV-

1. Topics in Antiviral Medicine 2017, 24.

12. Park, J.; Sayer, J.; Aniana, A.; Yu, X.; Weber, I.; Harrison, R.; Louis, J. Binding of clinical inhibitors to a model precursor of a rationally selected multidrug resistant HIV1 protease is significantly weaker than that to the released mature enzyme.

Biochemistry 2016, 55, 2390-2400.

13. Yu, Y.; Wang, J.; Shao, Q.; Shi, J.; Zhu, W. Effects of drug-resistant mutations on the dynamic properties of HIV-1 protease and inhibition by Amprenavir and Darunavir. Sci Rep 2015, 5, 10517.

14. Kletenkov, K.; Hoffman, D.; Böni, J.; Yerly, S.; Aubert, V.; Schöni-Affolter, F.; Struck, D.; Verheyen, J.; Klimkait, T.; Study, o.b.o.t.S.H.C. Role of Gag mutations in PI 
resistance in the Swiss HIV cohort study: bystanders or contributors? Journal of Antimicrobial Chemotherapy 2016, 72, 866-875.

15. Flynn, W.F.; Chang, M.W.; Tan, Z.; Oliveira, G.; Yuan, J.; Okulicz, J.F.; Torbett, B.E.; Levy, R.M. Deep sequencing of Protease Inhibitor resistant HIV patient isolates reveals patterns of correlated mutations in Gag and Protease. PLoS Comput Biol 2015, 11, e1004249, doi:10.1371/journal.pcbi.1004249.

16. Ghosn, J.; Delaugerre, C.; Flandre, P.; Galimand, J.; Cohen-Codar, I.; Raffi, F.; Delfraissy, J.-F.; Rouzioux, C.; Chaix, M. Polymorphism in Gag gene cleavage sites of HIV-1 non-B subtype and virological outcome of a first-line lopinavir/ritonavir single drug regimen. PLOS ONE 2011, 6, e24798, doi:10.1371/journal.pone.0024798.

17. Dam, E.; Quercia, R.; Glass, B.; Descamps, D.; Launay, O.; Duval, X.; Krausslich, H.-G.; Hance, A.J.; Clavel, F.; Group, A.S. Gag mutations strongly contribute to HIV1 resistance to Protease Inhibitors in highly drug-experienced patients besides compensating for fitness loss. Plos Pathogen 2009, 5, e1000345.

18. Aoki, M.; Venzon, D.J.; Koh, Y.; Aoki-Ogata, H.; Miyakawa, T.; Yoshimura, K.; Maeda, K.; Mitsuya, H. Non-cleavage site Gag mutations in Amprenavir-resisitant Human Immunodeficiency Virus Type 1 (HIV-1) predispose HIV-1 to rapid acquisition of Amprenavir resistance by delay development of resistance to other Protease Inhibitors. Journal of Virology 2009, 83, 3059-3068.

19. Parry, C.M.; Kohli, A.; Boinett, C.J.; Towers, G.J.; McCormick, A.L.; Pillay, D. Gag Determinants of Fitness and Drug Susceptibility in Protease Inhibitor-Resistant Human Immunodeficiency Virus Type 1. Journal of Virology 2009, 83, 9094-9101.

20. Tamiya, S.; Mardy, S.; Kavlick, M.F.; Yoshimura, K.; Mitsuya, H. Amino acid insertions near Gag cleavage sites restore the otherwise compromised replication of human immunodeficiency virus type 1 variants resistant to Protease Inhibitors. $J$ Virol 2004, 78, 12030-12040.

21. Maguire, M.F.; Guinea, R.; Griffin, P.; Macmanus, S.; Elston, R.C.; Wolfram, J.; Richards, N.; Hanlon, M.H.; Porter, D.J.; Wrin, T., et al. Changes in Human Immunodeficiency Virus Type 1 Gag at Positions L449 and P453 Are Linked to I50V Protease Mutants In Vivo and Cause Reduction of Sensitivity to Amprenavir and Improved Viral Fitness In Vitro. Journal of Virology 2002, 76, 7398-7406.

22. Gatanaga, H.; Suzuki, Y.; Tsang, H.; Yoshimura, K.; Kavlick, M.K.; Nagashima, K.; Gorelick, R.J.; Mardy, S.; Tang, C.; Summers, M.F., et al. Amino Acid Substitutions in Gag Protein at Non-cleavage sites Are Indispensable for the Development of a high Multitude of HIV-1 Resistance against Protease Inhibitors. The journal of Biological Chemistry 2002, 277, 5952-5961.

23. Codoner, F.M.; Pena, R.; Blanch-Lombarte, O.; Jimenez-Moyano, E.; Pino, M.; Vollbretch, T.; Clotet, B.; Martinez-Picado, J.; Draenert, R.; Prado, J.G. Gag-protease coevolution analyses define novel structural surfaces in the HIV-1 matrix and capsid involved in resistance to Protease Inhibitors. Sci Rep 2017, 7, 3717, doi:10.1038/s41598-017-03260-4.

24. Clavel, F.; Mammano, F. Review: Role of Gag in HIV Resistance to Protease Inhibitors. Viruses 2010, 2, 1411-1426. 
25. Ozen, A.; Lin, K.-H.; Yilmaz, N.K.; Schiffer, C.A. Structural basis and distal effects of Gag substrate coevolution in drug resistance to HIV-1 protease. Proc. Natl. Acad. Sci. USA 2014, 111, 15993-15998.

26. Myint, L.; Matsuda, M.; Matsuda, Z.; Yokomaku, Y.; Chiba, T.; Okano, A.; Yamada, K.; Sugiura, W. Gag non-cleavage site mutations contribute to full recovery of viral fitness in protease inhibitor-resistant human immunodeficiency virus type 1. Antimicrob Agents Chemother 2004, 48, 444-452.

27. Freed, E.O. Mini review: HIV-1 Gag proteins: Diverse Functions in the Virus Life Cycle. Virology 1998, 251, 1-15.

28. Pettit, S.C.; Moody, M.D.; Wehbie, R.S.; Kaplan, A.H.; Nantermet, P.V.; Klein, C.A.; Swanstrom, R. The p2 domain of Human Immunodeficiency Virus Type $1 \mathrm{Gag}$ regulates sequential proteolytic processing and is required to produce fully infectious virions. Journal of Virology 1994, 68, 8017-8027.

29. Prabu-Jeyabalan, M.; Nalivaika, E.; Schiffer, C. Substrate shape determines specificity of recognition for HIV-1 protease: analysis of crystal structures of six substrate complexes. Structure 2002, 10, 369-381.

30. Fun, A.; Wensing, A.M.; Verheyen, J.; Nijhuis, M. Human Immunodeficiency Virus gag and protease: partners in resistance. Retrovirology 2012, 9.

31. Pettit, S.C.; Lindquist, J.N.; Kaplan, A.H.; Swanstrom, R. Processing sites in the human immunodeficiency virus type 1 (HIV-1) Gag-Pro-Pol precursor are cleaved by the viral protease at different rates. Retrovirology 2005, 2.

32. Crooks, G.; Hon, G.; Chandonia, J.; Brenner, S. WebLogo: A sequence logo generator. Genome Research 2004, 14, 1188-1190.

33. Schneider, T.; Stephens, R. Sequence Logos: A new way to display consensus sequences. Nucleic Acids Res 1990, 18, 6097-6100.

34. Tang, M.W.; Shafer, R.W. HIV-1 Antiretroviral Resistance: Scientific Principles and Clinical Applications. Drugs 2012, 72, e1-e25.

35. Wensing, A.M.J.; Maarseveen, N.M.v.; Nijhuis, M. Fifteen years of HIV Protease Inhibitors: raising the barrier to resistance. Antiviral Research 2010, 85, 59-74.

36. Fumero, E.; Podzamczer, D. New patterns of HIV-1 resistance during HAART. Clinical Microbiology and Infection 2003, 9, 1077-1084.

37. Loulergue, P.; Delaugerre, C.; Jullien, V.; Viard, J.-P. HIV Drug resistance on HAART despite an undetectable viral load. Current HIV Research 2011, 9, 623-624.

38. Fun, A.; Maarseveen, N.M.v.; Pokorna, J.; Maas, R.E.; Schipper, P.J.; Konvalinka, J.; Nijhuis, M. HIV-1 protease inhibitor mutations affect the development of HIV-1 resistance to the maturation inhibitor bevirimat. Retrovirology 2011, 8.

39. Poon, A.F.; Pond, S.L.K.; Richman, D.D.; Frost, S.D. Mapping Protease Inhibitor Resistance to HIV Type 1 sequence polymorphisma within patients. Journal of Virology 2007, 81, 13598-13607. 
40. Blanco, J.-L.; Varghese, V.; Rhee, S.-Y.; Gatell, J.M.; Shafer, R.W. HIV-1 Integrase Inhibitor Resistance and its Clinical Implications. The journal of Infectious Diseases 2011, 203, 1204-1214.

41. Mitsuya, H.; Maeda, K.; Das, D.; Ghosh, A. Development of protease inhibitors and the fight with drug-resistant HIV-1 variants. Advances in Pharmacology 2008, 56, 169-197.

42. Gallant, J.E. Initial therapy of HIV Infection. Journal of Clinical Virology 2002, 25, 317-333.

43. Barrie, K.; Perez, E.; Lamers, S.; Farmerie, W.; Dunn, B.; Sleasman, J.; Goodenow, M. Natural variation in HIV-1 protease, Gag p7 and p6, and protease cleavage sites within gag/pol polyproteins: amino acid substitutions in the absence of protease inhibitors in mothers and children infected by human immunodeficiency virus type 1. Journal of Virology 1996, 219, 407-416.

44. Nakashima, M.; Ode, H.; Suzuki, K.; Fujino, M.; Maejima, M.; Kimura, Y.; Masaoka, T.; Hattori, J.; Matsuda, M.; Hachiya, A., et al. Unique flap conformation in an HIV-1 protease with high-level darunvir resistance. Front. Microbiol 2016, 7.

45. Appadurai, R.; Senapati, S. Dynamical network of HIV-1 protease mutants reveals the mechanism of drug resistance and unhindered activity. Biochemistry 2016, 55, 1529-1540.

46. Shen, C.; Chang, Y.; Agniswamy, J.; Harrison, R.; Weber, I. Conformational variation of an extreme drug resistant mutant of HIV protease. J Mol Graph Model 2015, 62, 87-96.

47. Ragland, D.A.; Nalivaika, E.A.; Nalam, M.N.; Prachanronarong, K.L.; Cao, H.; Bandaranayake, R.M.; Cai, Y.; Kurt-Yilmaz, N.; Schiffer, C.A. Drug Resistance conferred by mutations outside the active site through alterations in the dynamic and structural ensemble of HIV-1 protease. J. Am. Chem. Soc. 2014, 136, 11956-11963.

48. JD, C.; Gonzales, E.; Huang, X.; Smith, A.; de Vera, I.; D'Amore, P.; Rocca, J.; Goodenow, M.; Dunn, B.; Fanucci, G. Effects of PRE and POST therapy drugpressure selected mutations on HIV-1 protease conformational sampling. FEBS Letters 2014, 588, 3123-3128.

49. Huang, X.; Britto, M.; Kear-Scott, J.; Boone, C.; Rocca, J.; Simmerling, C.; McKenna, R.; Bieri, M.; Gooley, P.; Dunn, B., et al. The role of select subtype polymorphisms on HIV-1 protease conformational sampling and dynamics. Journal of Biological Chemistry 2014, 289, 17203-17214.

50. Duan, R.; Lazim, R.; Zhang, D. Understanding the basis of I50V-induced affinity decrease in HIV-1 protease via molecular dynamics simulations using polarized force field. Journal of Computational Chemistry 2015, 36, 1885-1892.

51. Antunes, D.; Rigo, M.; Sinigaglia, M.; de Medeiros, R.; Junqueira, D.; SEM, A.; Vieira, G. New insights into the in silico prediction of HIV protease resistance to nelfinavir. PLOS ONE 2014, 9, e87520.

52. Gupta, A.; Jamal, S.; Gopal, S.; Jain, R.; Wahi, D.; Grover, A. Structural studies on molecular mechanisms of Nelfinavir resistance caused by non-active mutation V77I in HIV-1 protease. BMC Bioinformatics 2015, 16, S10. 
53. Gao, F.; Robertson, D.; Carruthers, C.; Morrison, S.; Jian, B.; Chen; Barre-Sinoussi, F.; Girard, M.; Srinivasan, A.; Abimiku, A., et al. A comprehensive panel of near-full length clones and references sequences for non-subtype $B$ isolates of Human Immunodeficiency Virus Type 1. Journal of Virology 1998, 72, 5680-5698.

54. Martinez-Picado, J.; Savara, A.V.; Sutton, L.; D'Aquila, R.T. Replicative Fitness of Protease Inhibitor-Resistant Mutants of Human Immunodefieciency Virus Type 1. Journal of Virology 1999, 73, 3744-3752.

55. Bally, F.; Martinez, R.; Peters, S.; Sudre, P.; Telenti, A. Polymorphism of HIV Type 1 Gag p7/p1 and p1/p6 cleavage sites: clinical significance and implications for resistance to protease inhibitors. AIDS Research and Human Retroviruses 2000, 16, 1209-1213.

56. Bally, F.; Martinez, R.; Peters, S.; Sudre, P.; Telenti, A. Polymorphism of HIV Type 1 Gag p7/p1 and p1/p6 cleavage sites: clinical significance and implications for resistance to protease inhibitors. AIDS Research and Human Retroviruses 2004, 16.

57. Li, G.; Verheyen, J.; Rhee, S.-Y.; Voet, A.; Vandamme, A.-M.; Theys, K. Functional conservation of HIV-1 Gag: implications for rational drug design. Retrovirology 2013, 10, 126, doi:10.1186/1742-4690-10-126.

58. Yap, P.; Koh, D.W.S.; Su, C.T.T.; Chan, K.F.; Gan, S.K.E. Predicting mutations in HIV-1 Gag: Insight from in silico and an in vitro BSL2 platform on thermostability and allosteric effect. bioRXiv 2019, 10.1101/679852, doi:10.1101/679852.

59. Geller, R.; Domingo-Calap, P.; Cuevas, J.M.; Rossolillo, P.; Negroni, M.; Sanjuán, R. The external domains of the HIV-1 envelope are a mutational cold spot. Nature Communications 2015, 6, doi:10.1038/ncomms9571

60. Abram, M.E.; Ferris, A.L.; Shao, W.; Alvord, W.G.; Hughes, S.H. Nature, position, and frequency of mutations made in a single cycle of HIV-1 replication. Journal of Virology 2010, 84, 9864-9878, doi:10.1128/JVI.00915-10.

61. Su, C.T.T.; Kwoh, C.K.; Verma, C.S.; Gan, S.K.E. Modeling the full length HIV-1 Gag polyprotein reveals the role of its $\mathrm{p} 6$ subunit in viral maturation and the effect of noncleavage site mutations in protease drug resistance. Journal of Biomolecular Structure and Dynamics 2017, doi: 10.1080/07391102.2017.1417160, doi:doi: 10.1080/07391102.2017.1417160.

62. Doyon, L.; Croteau, G.; Thibeault, D.; Poulin, F.; Pilote, L.; Lamarre, D. Second locus involved in Human Immunodefiency Virus type 1 Resistance to Protease Inhibitors. Journal of Virology 1996, 70, 3763-3769.

63. Malet, I.; Roquebert, B.; Dalban, C.; Wirden, M.; Amellal, B.; Agher, R.; Simon, A.; Katlama, C.; Costagliola, D.; Calvez, V., et al. Association of Gag cleavage sites to Protease mutations and to virological response in HIV-1 treated patients. Journal of Infection 2007, 54, 367-374.

64. Parry, C.M.; Kolli, M.; Myers, R.; Cane, P.A.; Schiffer, C.; Pillay, D. Three residues in HIV-1 matrix contribute to protease inhibitor susceptibility and replication capacity. Antimicrob Agents Chemother 2011, 55, 1106-1113. 
65. Su, C.T.T.; Lua, W.H.; Ling, W.L.; Gan, S.K.E. Structural analyses of 2015-updated drug-resistant mutations in HIV-1 protease: an implication of protease inhibitor crossresistance. BMC Bioinformatics 2016, 17, 500.

66. Rhee, S.-Y.; Taylor, J.; Fessel, W.J.; Kaufman, D.; Towner, W.; Troia, P.; Ruane, P.; Hellinger, J.; Shirvani, V.; Zolopa, A., et al. HIV-1 Protease Mutations and Protease Inhibitor Cross-Resistance. Antimicrob Agents Chemother 2010, 54, 4253-4261, doi:10.1128/AAC.00574-10.

67. Ali, A.; Bandaranayake, R.M.; Cai, Y.; King, N.M.; Kolli, M.; Mittal, S.; Murzycki, J.F.; Nalam, M.N.; Nalivaika, E.A.; Ozen, A., et al. Molecular basis for drug resistance in HIV-1 Protease. Viruses 2010, 2, 2509-2535, doi:10.3390/v2112509.

68. Voshavar, C. Protease inhibitors for the treatment of HIV/AIDS: recent advances and future challenges. Curr Top Med Chem 2019, 10.2174/1568026619666190619115243, doi:10.2174/1568026619666190619115243.

69. Cohen, M.S.; Chen, Y.Q.; McCauley, M.; Gamble, T.; Hosseinipour, M.C.; Kumarasamy, N.; Hakim, J.G.; Kumwenda, J.; Grinsztejn, B.; Pilotto, J.H.S., et al. Prevention of HIV-1 Infection with Early Antiretroviral Therapy. The New England Journal of Medicine 2011, 365.

70. Mehellou, Y.; Clercq, E.D. Twenty-six years of Anti-HIV Drug discovery: where do we stand and where do we go? Journal of Medicinal Chemistry 2009, 10.1021/jm900492g, doi:10.1021/jm900492g.

71. Perez, D.; Azoulay, D. Anti-DFS70 autoantibodies in HIV-1 positive individuals. Current Opinion in Rheumatology 2018, 30, 361-364, doi:10.1097/BOR.000000000000049.

72. Kwong, P.; Mascola, J.; Nabel, G.J. Broadly neutralizing antibodies and the search for an HIV-1 vaccine: the end of the beginning. Nat Rev Immunol 2013, 13, 693-701, doi:10.1038/nri3516.

73. Bird, G.; Irimia, A.; Ofek, G.; Kwong, P.; Wilson, I.; Walensky, L. Stapled HIV-1 peptides recapitulate antigenic structures and engage broadly neutralizing antibodies. Nat Struct Mol Biol. 2014, 21, 1058-1067.

74. Deng, K.; Pertea, M.; Rongvaux, A.; Wang, L.; Durand, C.M.; Ghiaur, G.; Lai, J.; McHugh, H.L.; Hao, H.; Zhang, H., et al. Broad CTL response is required to clear latent HIV-1 due to dominance of escape mutations. Nature 2015, 517, 381-385, doi:10.1038/nature14053.

75. Martin, D.; Salzwedel, K.; Allaway, G. Berivimat: a novel maturation inhibitor for the treatment of HIV-1 infection. Antivir Chem Chemother 2008, 19, 107-113.

76. Thenin-Houssier, S.; Valente, S.T. HIV-1 capsid inhibitors as antiretroviral agents. Curr HIV Res 2016, 14, 270-282.

77. Nguyen, A.T.; Feasley, C.L.; Jackson, K.W.; Nitz, T.J.; Salzwedel, K.; Air, G.M.; Sakalian, M. The prototype HIV-1 maturation inhibitor, berivimat, binds to the CASP1 cleavage site in immature Gag particles. Retrovirology 2011, 8, 101, doi:10.1186/1742-4690-8-101. 
78. Purdy, M.; Shi, D.; Chrustowicz, J.; Hattne, J.; Gonen, T.; Yeager, M. MicroED structures of HIV-1 Gag CTD-SP1 reveal binding interactions with the maturation inhibitor berivimat. Proc Natl Acad Sci USA 2018, 115, 13258-13263, doi:10.1073/pnas.1806806115.

79. Keller, P.W.; Adamson, C.S.; Heymann, J.B.; Freed, E.O.; Steven, A.C. HIV-1 maturation inhibitor Berivimat stabilizes the immature Gag lattice. Journal of Virology 2011, 85, 1420-1428, doi:10.1128/JVI.01926-10.

80. Dang, Z.; Qian, K.; Ho, P.; Zhu, L.; Lee, K.-H.; Huang, L.; Chen, C.-H. Synthesis of betulinic acid derivatives as entry inhibitors against HIV-1 and Berivimat-resistant HIV-1 variants. Bioorg Med Chem Lett 2012, 22, 5190-5194.

81. Seclen, E.; Gonzalez, M.M.; Corral, A.; de Mendoza, C.; Soriano, V.; Poveda, E. High prevalence of natural polymorphism in Gag (CA-SP1) associated with reduced response to Berivimat, an HIV-1 maturation inhibitor. AIDS 2010, 24, 467-469, doi:10.1097/QAD.0b013e328335ce07.

82. Margot, N.A.; Gibbs, C.S.; Miller, M.D. Phenotypic susceptibility to Berivimat in isolates from HIV-1 infected patients without prior exposure to Berivimat. Antimicrob Agents Chemother 2010, 54, 2345-2353, doi:10.1128/AAC.01784-09.

83. Verheyen, J.; Verhofstede, C.; Knops, E.; Vandekerckhove, L.; Fun, A.; Brunen, D.; Dauwe, K.; Wensing, A.M.; Pfister, H.; Kaiser, R., et al. High prevalence of Berivimat resistance mutations in protease inhibitor-resistant HIV isolates. AIDS 2010, 24, 669673, doi:10.1097/QAD.0b013e32833160fa.

84. Ray, N.; Li, T.; Lin, Z.; Protack, T.; van Ham, P.; Hwang, C.; Krystal, M.; Nijhuis, M.; Lataillade, M.; Dicker, I. The second-generation maturation inhibitor GSK3532795 maintains potent activity toward HIV Protease Inhibitor-resistant clinical isolates. $J$ Acquir Immune Defic Syndr 2017, 75, 52-60, doi:10.1097/QAI.0000000000001304.

85. Morales-Ramirez, J.; Bogner, J.R.; Molina, J.-M.; Lombaard, J.; Dicker, I.B.; Stock, D.A.; DeGrosky, M.; Gartland, M.; Dumitrescu, T.P.; Min, S., et al. Safety, efficacy, and dose response of the maturation inhibitor GSK3532795 (formerly known as BMS-955176) plus tenofovir/emtricitabine once daily in treatment-naive HIV-1infected adults: Week 24 primary analysis from a randomized Phase Ilb trial. PLoS ONE 2018, 13, e0205368.

86. Phua, S.X.; Chan, K.F.; Su, C.T.T.; Poh, J.J.; Gan, S.K.E. Perspective: The promises of a holistic view of proteins - impact on antibody engineering and drug discovery. Biosci Rep 2019, 39, pii: BSR20181958, doi:10.1042/BSR20181958.

87. Chiang, R.Z.H.; Gan, S.K.E.; Su, C.T.T. A computational study for rational HIV-1 non-nucleoside reverse transcriptase inhibitor selection and the discovery of novel allosteric pockets for inhibitor design. Bioscience Reports 2018, 38, BSR20171113.

88. Chan, K.F.; Phua, S.X.; Su, C.T.T.; Gan, S.K.E. Inhibiting HIV-1 and MMLV Reverse Transcriptase: The potential of an allosteric broad-spectrum inhibitor. bioRXiv 2019, 10.1101/699470, doi:10.1101/699470.

89. Richard, S.; Selle, F.; Lotz, J.; Khalil, A.; Gligorov, J.; Soares, D. Pertuzumab and Trastuzumab: the rationale way to synergy. An Acad Bras Cienc 2016, 88, 565-577, doi:10.1590/0001-3765201620150178. 
90. Capelan, M.; Pugliano, L.; De Azambuja, E.; Bozovic, I.; Saini, K.; Sotiriou, C.; Loi, S.; Piccart-Gebhart, M. Pertuzumab: new hope for patients with HER2-positive breast cancer. Annals of Oncology 2012, 0, 1-10, doi:10.1093/annonc/mds328.

91. Maldonado, J.O.; Martin, J.L.; Mueller, J.D.; Zhang, W.; Mansky, L.M. New insight into retroviral Gag-Gag and Gag-membrane interactions. Front Microbiol 2014, 5, 302, doi:10.3389/fmicb.2014.00302.

92. El Meshri, S.; Dujardin, D.; Godet, J.; Richert, L.; Boudier, C.; Darlix, J.; Didier, P.; Mely, Y.; de Rocquigny, $\mathrm{H}$. Role of the nucleocapsid domain in HIV-1 Gag oligomerization and trafficking to the plasma membrane: a fluorescence lifetime imaging microscopy investigation. J Mol Biol 2015, 427, 1480-1494, doi:10.1016/j.jmb.2015.01.015.

93. Martin, J.L.; Mendonca, L.M.; Angert, I.; Mueller, J.D.; Zhang, W.; Mansky, L.M. Disparate contributions of human retrovirus capsid subdomains to Gag-Gag oligomerization, virus morphology, and particle biogenesis. Journal of Virology 2017, 91, e00298-00217, doi:10.1128/JVI.00298-17. 
Table 1: Gag and Protease paired mutations compensating for viral fitness and viral replication

\begin{tabular}{|c|c|c|c|}
\hline & & $\begin{array}{lllll}363 & 378 & 432 & 441 & 500\end{array}$ & \\
\hline Inhibitor & Strain or lab clone & Mutations on Gag & Mutations on Protease \\
\hline Amprenavir & HIV-1 NL4-3 (pNL4-3) & V35I-L75R-H219Q & L10F-V32I-M46I-I84V \\
\hline Amprenavir & HIV-1 NL4-3 (pNL4-3) & L75R-H219Q-R409K-L449F-E468K & L10F-V32I-M46I-I84V \\
\hline Amprenavir & HIV-1 NL4-3 (pNL4-3) & E12K-V35I-L75R-H219Q-V390D-R409K-L449F-E468K & L10F-V32I-M46I-I54M-A71V-I84V \\
\hline JE-2147 & HIV-1 NL4-3 (pNL4-3) & H219Q-V390D & M46I-I84V \\
\hline JE-2147 & HIV-1 NL4-3 (pNL4-3) & H219Q-V390D-R409K-L449F & V32I-M46I-I47V-V82I-I84V \\
\hline KNI-272 & HIV-1 NL4-3 (pNL4-3) & V35I-E40K-G123E-H219Q-G381S-R409K-A431V & V32I-M46I-A71V-V82I-I84V \\
\hline UIC-94003 & HIV-1 NL4-3 (pNL4-3) & E12K-E40K-G123E-Q199H-H219Q-R409K-G412D-L449F-E468K & L10F-M46I-I50V-A71V \\
\hline Amprenavir & HIV-1 HXB2 & P453L & I50V \\
\hline BILA-1906BS & HIV-1 strain IIIB & L449F & M46L-A71V-184V \\
\hline BILA-2185BS & HIV-1 strain IIIB & L449F-Q430R-A431V & L23I-V32I-M46I-I47V-I54M-A71V-I84V \\
\hline Indinavir & HIV-1 pNL4.3 & $\mathrm{A} 431 \mathrm{~V}-1437 \mathrm{~V}$ & V82A \\
\hline Ritonavir/Saquinavir & HIV-1 subtype $B^{\#}$ & A431V-L449F & $184 \mathrm{~V}$ \\
\hline
\end{tabular}

\# the study involves patients 
